\title{
Barriers for a wider use of left atrial appendage occlusion as an alternative to oral anticoagulation
}

\section{Introduction}

For many years we haven't seen significant improvements in the treatment of hyperlipidemia, with ezetimibe rendering modest results over statin treatment and failure of cholesteryl ester transfer protein (CETP) inhibitors as torcetrapib, dalcetrapib and evacetrapib. Proprotein convertase subtilisin/kexin type 9 (PCSK9) inhibitors finally offered good news in this field. The main caveat of these drugs is the price, up to $\$ 14,000$ /year in USA but not less than $\$ 6,000$ in Europe. The estimated incremental cost-effectiveness ratio (ICER; cost per quality-adjusted life-year QALY) over statin + ezetimibe treatment in heterozygous familial hypercholesterolemia has been calculated in $\$ 503,000$; ICER per QALY of this treatment on top of statin treatment in patients with atherosclerotic cardiovascular disease is $\$ 414,000 .^{1}$

In any case, these are values far from the accepted willingnessto-pay thresholds. For PCSK9 inhibitor therapy to reach an ICER of $\$ 100,000$ per QALY, the annual cost of drug treatment should be $\$ 4,536$; if the aim is the most used ICER of $\$ 50,000$ per QALY, the annual cost should drop to $\$ 2,261$. Despite these data, you could bet on that these drugs are the next blockbusters. Maybe they need some adjustments in price, but some of their advantages (1 single dose every 2-4weeks!) overcome many of their limitations.

A clear question arises: why a cheaper, effective, treatment as left atrial appendage closure (LAAC) for non-valvular atrial fibrillation (NVAF) couldn't reach a broader use? The cost of the device for LAAC + procedure itself in Germany is estimated in $€ 9,136,{ }^{2}$ slightly superior to 1-year treatment with PCSK9 inhibitors. ${ }^{1}$ The figures are similar in USA: the cost for LAAC procedure +2 transesophageal echocardiograms is $\$ 16,109 .{ }^{3}$

\section{Barriers for a widespread use of LAAC}

In 2012, the Scientific Initiative Committee of the European Heart Rhythm Association (EHRA) conducted a survey in 36 centers to assess clinical practice in relation to the use of LAAC. ${ }^{4}$ Although it was conducted before the availability of relevant results of LAAC, some interesting conclusions were drawn. The main barriers described by the participants in the survey ( $67 \%$ of whom were already performing LAAC procedures) are discussed herein.

\section{Technical difficulty of the procedure}

Although subjective issues can hardly be analyzed, this is one the limitations that should improve over time. Also, it could be explained by the fact that this procedure is performed by a wide variety of specialists, not only by electrophisiologists and interventional cardiologists. The wider use of different transeptal procedures, as atrial fibrillation ablation techniques, mitral valve procedures, perivalvular leak closure, ... may help in solving this potential

\author{
Volume 6 Issue 4 - 2016 \\ Armando Perez de Prado, Carlos Cuellas \\ Ramon, Rodrigo Estevez Loureiro, Maria \\ Lopez Benito, Tomas Benito Gonzalez, Felipe \\ Fernandez Vazquez \\ Department of Cardiology, Hospital Universitario de Leon, \\ Spian \\ Correspondence: Armando Pérez de Prado, Department of \\ Cardiology, Hospital Universitario de León. Altos de Nava SN, \\ 24008, León, Spain, Tel +34 987237683 \\ Email aperez@secardiologia.es
}

Received: August 21, 2016 | Published: October 03, 2016

problem. Nevertheless, it was considered the less important obstacle in the EHRA survey. ${ }^{4}$

\section{Risk of complications}

This is a key obstacle that has stopped the widespread use of these techniques for many years. The first data available from a randomized trial (PROTECT $\mathrm{AF}^{5}$ ) on the safety and efficacy of the LAAC procedure with the Watchman ${ }^{\circledR}$ device (Boston Scientific) showed a procedure-related rate of complications of $7.7 \%$. The main components of this safety endpoint are pericardial effusion (and cardiac tamponade), procedure related stroke (less than $1 \%$ ) and device thrombosis. Further analysis ${ }^{6}$ demonstrated a "learning curve" effect: first half of the PROTECT AF patients showed almost a double rate of complications than the second half; patients from CAP registry, ${ }^{6}$ from centers and operators with extended experience, showed a $3.7 \%$ complications rate.

The LAAC procedure has been successfully performed using the Amplatzer Cardiac Plug $(\mathrm{ACP} \circledast)$ and its second version, Amulet ${ }^{\circledR}$, devices (St Jude Medical). The complication rate ${ }^{7}$ is quite similar to that described for the Watchman ${ }^{\circledR}$. In this case, the embolization rate seems to be slightly superior $(2 \%)$. Careful procedures and some tips ${ }^{8}$ can help in shortening the time span of the learning curves.

\section{Limited efficacy data for stroke prevention}

It should be remarked that this was the second main obstacle perceived by operators in 2012, before the PROTECT AF long-term, ${ }^{9}$ PREVAIL $^{10}$ and ASAP ${ }^{11}$ results were published. All of them confirmed the superiority of LAAC procedure over warfarin treatment. In 2012, the last update of the Clinical Guidelines on Atrial Fibrillation of the European Society of Cardiology (ESC) ${ }^{12}$ was also published. The indication for the LAAC was established as Class IIb, Level of Evidence B (supporting evidence ${ }^{5,6}$ ): it "may be considered in patients with a high stroke risk and contraindications for long-term oral anticoagulation". This indication has been broadly contested as, thus 
far, no evidence was available on the efficacy of LAAC for that kind of patients. It was one year after when the ASAP study ${ }^{11}$ demonstrated the safety and efficacy of LAAC in patients with a contraindication for oral anticoagulation (OAC). The new version of the ESC Clinical Guidelines on Atrial Fibrillation is awaited at the upcoming ESC 2016 Meeting at Rome and changes in this field are expected.

By this time, at least 4 novel oral anticoagulants (NOAC) have been approved for the treatment of NVAF. Apixaban seems to offer the best results versus warfarin with a hazard ratio (HR) $0.79,0.33 \%$ absolute risk reduction (ARR), in the primary efficacy outcome and a HR $0.69,0.96 \%$ ARR, in the rate of major bleeding. ${ }^{13}$ Also, apixaban is the only NOAC that has been tested in patients in whom vitamin $\mathrm{K}$ antagonist therapy was "unsuitable". ${ }^{14}$ The study was prematurely terminated because apixaban was clearly superior to aspirin in terms of efficacy (HR 0.45) with a slight increase in major bleeding (HR 1.13). It should be emphasized that most of the patients included don't want to take vitamin $\mathrm{K}$ antagonists (37\%), have a low CHADS2 score $<1(21 \%)$ or were unable or considered unlikely to assess the INR measurements at the requested intervals (43\%). Only 3\% of the patients have had a serious bleeding while in OAC therapy. No information on the HAS-BLED score of the patients is supplied. The comparison with similar studies of LAAC devices is, then, difficult, as the profile of the patients seems to be quite different.

Despite all the available evidence, many physicians still cast doubts on LAAC efficacy and prefer the use of NOACs in this kind of patients. It is expected that the doubts will disappear with the upcoming results of ongoing trials as Left Atrial Appendage Closure (with Watchman ${ }^{\circledR}$ or Amulet ${ }^{\circledR}$ ) vs. Novel Anticoagulation Agents in Atrial Fibrillation (PRAGUE-17 - NCT02426944), Prevention of Stroke by Left Atrial Appendage Closure in Atrial Fibrillation Patients After Intracerebral Hemorrhage (comparing Amulet ${ }^{\circledR}$ with (N)OAC - NCT02830152), and Evaluation of Watchman ${ }^{\circledR}$ Left Atrial Appendage Occlusion Device in Patients With Atrial Fibrillation Versus Rivaroxaban (NCT02549963).

\section{Price / reimbursement issues}

This was acknowledged as the main obstacle for a widespread LAAC use in the EHRA survey. ${ }^{4}$ However, it should be the weaker argument against LAAC. Different studies have demonstrated the cost-effectiveness of the LAAC procedure for NVAF., ${ }^{2,3,15}$ What's more, all of them have demonstrated its superiority over the NOACs. Previous analysis demonstrated that NOACs are cost-effective as compared with Warfarin, ${ }^{16}$ although some authors ${ }^{17}$ sustain that only apixaban is really cost-effective below the threshold of $\$ 50,000$ (ICER per QALY gained, \$25,816). The ICER per QALY gained of LAAC over warfarin has been calculated as low as $\$ 3,432 .{ }^{15}$ A very interesting study ${ }^{3}$ determined the time to cost-effectiveness and the dominance (less cost with more efficacy) of the different treatments for NVAF. The main results are:

a. As compared with warfarin, LAAC is cost-effective at 7years (\$42,99 per QALY).

b. As compared with warfarin, NOACs are cost-effective at 16years (\$48,446/QALY).

c. LAAC is dominant over NOACs at 5 years and warfarin at 10years.

d. At 10 years, LAAC provide more QALYs than warfarin and NOACs.

e. The same group of investigators performed a similar analysis ${ }^{2}$ in patients with absolute contraindications to warfarin. The selected comparators for the LAAC were aspirin plus clopidogre ${ }^{18}$ and apixaban. ${ }^{14}$ The results were parallel to the previous:

f. At 5years, LAAC is cost effective compared with aspirin + clopidogrel with an ICER of $€ 16,971$.

g. At 5years, LAAC is cost effective compared with apixaban at 7 years with an ICER of $€ 9,040$.

h. LAAC is dominant over both aspirin + clopidogrel and apixaban at 8years.

\section{Conclusion}

Left atrial appendage closure is a well-established procedure. Data from randomized trials and observational studies support its efficacy over oral anticoagulation treatment in the management of patients with non-valvular atrial fibrillation. Upcoming results from studies comparing this procedure directly with novel oral anticoagulants should strengthen its value. Cost-effectiveness analyses render very favorable results for this procedure, saving costs with higher efficacy.

PS: While the paper was being processed, the ESC 2016 update of guidelines for the management was released. ${ }^{19}$ Unexpectedly, the recommendation for LACC remained in a class IIb, level of evidence B (LAA occlusion may be considered for stroke prevention in patients with $\mathrm{AF}$ and contra-indications for long-term anticoagulant treatment (e.g. those with a previous life-threatening bleed without a reversible cause).

\section{Acknowledgments}

None.

\section{Conflicts of interest}

Author declares there are no conflicts of interest.

\section{Funding}

None.

\section{References}

1. Kazi DS, Moran AE, Coxson PG, et al. Cost-effectiveness of PCSK9 Inhibitor Therapy in Patients With Heterozygous Familial Hypercholesterolemia or Atherosclerotic Cardiovascular Disease. JAMA .2016;316(7):743-753.

2. Reddy VY, Akehurst RL, Armstrong SO, et al. Cost effectiveness of left atrial appendage closure with the Watchman device for atrial fibrillation patients with absolute contraindications to warfarin. Europace. 2016;18(7):979-986.

3. Reddy VY, Akehurst RL, Armstrong SO, et al. Time to Cost-Effectiveness Following Stroke Reduction Strategies in AF: Warfarin Versus NOACs Versus LAA Closure. J Am Coll Cardiol. 2015;66(24):2728-2739.

4. Lip GY, Dagres N, Proclemer A, et al. Left atrial appendage occlusion for stroke prevention in atrial fibrillation in Europe: results of the European Heart Rhythm Association survey. Europace. 2013;15(1):141-143.

5. Holmes DR, Reddy VY, Turi ZG, et al. Percutaneous closure of the left atrial appendage versus warfarin therapy for prevention of stroke in patients with atrial fibrillation: a randomised non-inferiority trial. Lancet. 2009;374(9689):534-542.

6. Reddy VY, Holmes D, Doshi SK, et al. Safety of percutaneous left atrial appendage closure: results from the Watchman Left Atrial Appendage System for Embolic Protection in Patients with AF (PROTECT AF) clinical trial and the Continued Access Registry. Circulation. $2011 ; 123(4): 417-424$. 
7. Park JW, Bethencourt A, Sievert H, et al. Left atrial appendage closure with Amplatzer cardiac plug in atrial fibrillation: initial European experience. Catheter Cardiovasc Interv . 2011;77(5):700-706.

8. Tzikas A, Gafoor S, Meerkin D, et al. Left atrial appendage occlusion with the AMPLATZER Amulet device: an expert consensus step-by-step approach. EuroIntervention . 2016;11(13):1512-1521.

9. Reddy VY, Sievert H, Halperin J, et al. Percutaneous left atrial appendage closure vs warfarin for atrial fibrillation: a randomized clinical trial. JAMA. 2014;312(19):1988-1998.

10. Holmes DR, Jr., Kar S, Price MJ, et al. Prospective randomized evaluation of the Watchman Left Atrial Appendage Closure device in patients with atrial fibrillation versus long-term warfarin therapy: the PREVAIL trial. J Am Coll Cardiol. 2014;64(1):1-12.

11. Reddy VY, Mobius-Winkler S, Miller MA, et al. Left atrial appendage closure with the Watchman device in patients with a contraindication for oral anticoagulation: the ASAP study (ASA Plavix Feasibility Study With Watchman Left Atrial Appendage Closure Technology). J Am Coll Cardiol. 2013;61(25):2551-2556.

12. Camm AJ, Lip GY, De Caterina R, et al. 2012 focused update of the ESC Guidelines for the management of atrial fibrillation: an update of the 2010 ESC Guidelines for the management of atrial fibrillation. Developed with the special contribution of the European Heart Rhythm Association. Eur Heart J . 2012;33(21):2719-2747.

13. Granger CB, Alexander JH, McMurray JJ, et al. Apixaban versus warfarin in patients with atrial fibrillation. $N$ Engl J Med. 2011;365(11):981-992.
14. Connolly SJ, Eikelboom J, Joyner C, et al. Apixaban in patients with atrial fibrillation. $N$ Engl J Med . 2011;364(9):806-817.

15. Lee VW, Yan B, Chow I, et al. Cost-effectiveness Analysis Of Left Atrial Appendage Occlusion Device Compared With 7 Oral Anticoagulants For Stroke Prevention In Atrial Fibrillation (Abst). J Am Coll Cardiol . 2014;64(11 S)

16. Harrington AR, Armstrong EP, Nolan PE, et al. Cost-effectiveness of apixaban, dabigatran, rivaroxaban, and warfarin for stroke prevention in atrial fibrillation. Stroke . 2013;44(6):1676-1681.

17. Shah A, Shewale A, Hayes CJ, et al. Cost-Effectiveness of Oral Anticoagulants for Ischemic Stroke Prophylaxis Among Nonvalvular Atrial Fibrillation Patients. Stroke. 2016;47(6):1555-1561.

18. Investigators A, Connolly SJ, Pogue J, et al. Effect of clopidogrel added to aspirin in patients with atrial fibrillation. $N$ Engl J Med. 2009;360(20):2066-2078

19. Kirchhof P, Benussi S, Kotecha D, et al. 2016 ESC Guidelines for the management of atrial fibrillation developed in collaboration with EACTS: The Task Force for the management of atrial fibrillation of the European Society of Cardiology (ESC)Developed with the special contribution of the European Heart Rhythm Association (EHRA) of the ESCEndorsed by the European Stroke Organisation (ESO). Eur Heart J. 2016. 